\title{
ESTRATÉGIA ORTODÔNTICO-CIRÚRGICA EM RELAÇÃO AO ELEMENTO SUPRANUMERÁRIO, LOCALIZADO NA REGIÃO DO INCISIVO LATERAL SUPERIOR EM INDIVÍDUO JOVEM: RELATO DE CASO
}

\author{
Orthodontic-surgical strategy regarding the supernumerary element, located in \\ the upper lateral incisor region in young individual: case report
}

\section{Fabíola Villela Alves Pereira ${ }^{1}$ Maurício de Oliveira Moraes ${ }^{1}$ João Carlos Sacramento Conceição ${ }^{1}$ Wellington de Oliveira Vasques ${ }^{1}$}

${ }^{1}$ Centro Universitário de Valença (UNIFAA) Valença $(R J)$

Autor correspondente:

Fabíola Villela Alves Pereira E-mail: drafavillela@hotmail.com

\section{Como citar este artigo:}

PEREIRA, F.V.A. Estratégia ortodôntico-cirúrgica em relação ao elemento supranumérico, localizado na região do inciso lateral superior em indivíduo jovem: relato de caso. Revista Saber Digital, v. 14, n. 3, p. 69-82 2021.

Data de Submissão: 19/11/21

Data de aprovação: 16/12/21

Data de publicação: 21/12/21

\section{(c) (1) ()}

Esta obra está licenciada com uma licença http://creativecommons.org/licenses/by-nc/4.0/

\section{RESUMO}

Introdução: Dado como uma anomalia de número por superatividade da lâmina dentária, os dentes supranumerários têm sua origem devido a causas genéticas, estando presentes em displasias congênitas se apresentando nas mais diferentes formas, desde mal formado até normal, uni ou bilateral, na maxila e mandíbula. Em alguns casos, eles também veem de forma múltipla. Uma precoce e correta abordagem, em pacientes jovens, frente a uma alteração de desenvolvimento, visando a presença de dentes supranumerários modificando a oclusão, vem apresentando resultados satisfatórios e importantes, para manter a função e estética da cavidade bucal. Objetivo: Apresentar um caso clínico que trata de uma expansão rápida da maxila (ERM), feita inicialmente, em paciente portador de atresia maxilar e elemento supranumerário e realizar uma revisão da literatura acerca de estratégias ortodôntico-cirúrgicas em relação aos elementos supranumerários associados as más oclusões. Relato do caso: O presente artigo apresenta um caso clínico de um paciente jovem, 09 anos de idade, no qual, adotou-se procedimentos como o acompanhamento radiográfico, com radiografias periapicais e panorâmicas, e a expansão rápida da maxila. Por meio da expansão rápida da maxila objetivou-se induzir a irrupção passiva do supranumerário para uma exodontia simples na região do elemento dental 12. Conclusão: Foi decidido realizar a exodontia do supranumerário após a ERM, onde o elemento supranumerário não obliterava a linha de irrupção dos elementos permanentes. Com a irrupção desse elemento , foi efetivado um ato cirúrgico mais simples, diminuindo riscos e complicações ao paciente pediátrico, além de exposição aos raios $x$, com a realização de uma Tomografia Computadorizada.

Palavras-chave: Dentes Supranumerários; Estética Dentária; Expansão Rápida da Maxila.
ABSTRACT
Introduction: Given as a number anomaly due to the overactivity of the dental lamina, supernumerary teeth have their origin due to genetic causes, being present in congenital dysplasias presenting in the most different forms, from malformed to normal, uni or bilateral, in the maxilla and mandible. In some cases, they also come in multiple forms. An early and correct approach, in young patients, facing a developmental change, aiming at the presence of supernumerary teeth modifying the occlusion, has been showing satisfactory and important results, to maintain the function and aesthetics of the oral cavity. Objective: To present a clinical case that deals with rapid maxillary expansion (ERM), initially performed in a patient with maxillary atresia and a supernumerary element, and to carry out a literature review on orthodontic-surgical strategies in relation to supernumerary elements associated with malocclusions. Case report: This article presents a clinical case of a young patient, 09 years old, in which procedures were adopted such as radiographic follow-up, with periapical and panoramic radiographs, and rapid 
maxillary expansion. Through rapid maxillary expansion, the objective was to induce the passive eruption of the supernumerary for a simple extraction in the region of the dental element 12 . Conclusion: It was decided to carry out the extraction of the supernumerary after ERM, where the supernumerary element did not obliterate the irruption line of the permanent elements. With the irruption of this element, a simpler surgical procedure was carried out, reducing risks and complications for the pediatric patient, in addition to exposure to $\mathrm{x}$ rays, with the performance of a Computerized Tomography.

Keywords: Supernumerary Theeth; Dental Aesthetics, Dentist; Rapid Maxillary Expansion.

\section{INTRODUÇÃO}

É comum em tempos atuais na odontologia, principalmente na odontopediatria, o desenvolvimento de um trabalho visando à importância de uma boa saúde bucal, oferecendo um acompanhamento preventivo ao paciente dos primeiros dentes, uma melhor opção de tratamento (REIS et al., 2006). O acompanhamento é essencial, tanto para as alterações fisiológicas quanto as emocionais, onde são importantes as intervenções que, por vezes, é precoce, de acordo com as necessidades bucais do paciente, bem como com as alterações de desenvolvimento. A presença de dentes supranumerários excede qualquer cuidado preventivo, necessitando de uma abordagem curativa oportuna frente a cada caso (REIS et al., 2006; HENRIQUE et al., 2010).

Os supranumerários nada mais são que dentes que se desenvolvem além do número considerado normal, em uma arcada dentária. Em alguns casos, os supranumerários irrompem normalmente e, em outras situações, permanecem impactados. Podem assumir uma posição ectópica e muitas vezes estarem invertidos. Essa anomalia pode acontecer de várias maneiras, sendo elas, bilateralmente, unilateral, múltipla ou isolada e, em alguns casos, em ambos os arcos. Geralmente a população masculina é a mais acometida por esse fator (HENRIQUES et al., 2008).

Para um melhor entendimento sobre a formação de um dente supranumerário é fundamental o conhecimento do processo de desenvolvimento dentário. Dividida em fases, a embriologia dentária é composta pela iniciação, observada no feto a partir da 6 ${ }^{\mathrm{a}}$ semana, quando tem a inicialização da formação 
dos órgãos dentários e da lâmina dentária; pela proliferação, em que há uma multiplicação das células da fase de iniciação, provocando a formação do germe dentário; pela diferenciação histológica das células e uma organização delas para determinar a forma e o tamanho do dente; calcificação e oposição, que correspondem à formação da deposição mineral e a matriz. Possíveis alterações nas diferentes fases vão determinar distúrbios dentários (REIS et al., 2006).

A conduta do profissional perante casos de dentes supranumerários pode variar. Alguns sugerem a remoção precoce dos mesiodens, de forma que, a remoção impeça o aparecimento de algumas patologias alterando também a erupção dos dentes permanentes (REIS et al., 2006; VALARELLI et al., 2012). A remoção cirúrgica de um dente supranumerário é recomendada, quando este estiver interferindo na cronologia normal de erupção dos demais dentes adjacentes, podendo assim, realizar uma abordagem mais conservadora (HUANG, 1992).

Má oclusão ocasionada pela presença de um dente supranumerário pode ser desde um simples desvio de linha média, até o comprometimento de um dente definitivo, natural, em função de uma reabsorção radicular, levando a uma extração não desejada (MARCHETTI; OLIVEIRA, 2015).

Desse modo, o objetivo do presente trabalho foi apresentar um caso clínico que trata de uma expansão rápida da maxila (ERM), feita inicialmente, em paciente portador de atresia maxilar e elemento supranumerário e realizar uma revisão da literatura, acerca de estratégias ortodôntico-cirúrgicas em relação aos elementos supranumerários associados às más oclusões.

\section{RELATO DE CASO}

O relato de caso deste artigo é de um indivíduo do sexo masculino com as iniciais E. S. G. com idade de 9 anos e 5 meses no início do tratamento, que por meio de seu responsável, procurou consultório particular, no município do Rio de Janeiro, em busca de tratamento ortodôntico. A queixa principal era 
"dentes tortos, dentes que não nasceram". A figura 01 apresenta fotos da oclusão, anteriores ao tratamento.

Esse caso clínico teve aprovação no Comitê de Ética em Pesquisa em Seres Humanos do Centro Universitário de Valença (UniFAA), conforme Parecer Consubstanciado número: 4.529.872 (ANEXO):

Figura 1 - Fotos ao início do tratamento: A) vista lateral direita, B) vista frontal. C) vista lateral esquerda.

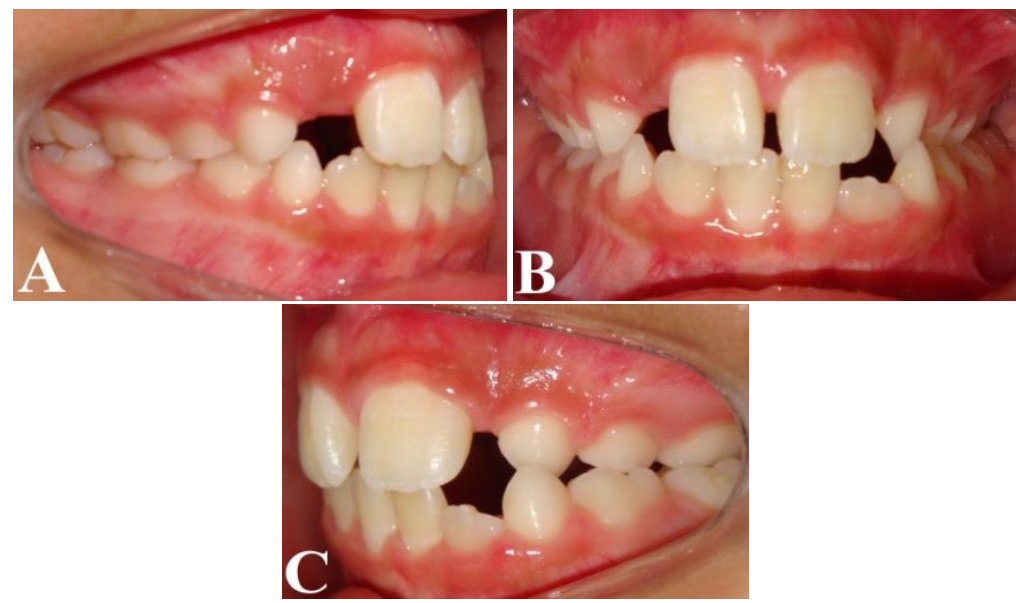

Fonte: Acervo pessoal.

A ficha clínica de anamnese foi minuciosamente preenchida, com todas as informações necessárias como, história médica e odontológica do paciente, assim como orientações sobre o tratamento. Um termo de consentimento foi assinado pela responsável do menor, que autorizava o uso de imagens relacionadas ao tratamento, na publicação do presente artigo. Em uma consulta inicial, após uma avaliação da documentação ortodôntica, através da radiografia panorâmica, foi constatada a presença de um elemento supranumerário na região do elemento 12 , como mostram as figuras 2 e 3 . A técnica de Clark foi realizada para definição da sua localização, que se mostrou pela palatina e acima da linha incisal do elemento 12. O exame de Tomografia Computadorizada (TC) não foi solicitado neste momento, com o objetivo de causar menos 
Estratégia ortodôntico-cirúrgica em relação ao elemento supranumérico, localizado na região do inciso lateral superior em indivíduo jovem: relato de caso

Pereira FVA

exposição do paciente à radiação, bem como, diminuir os custos, a pedido do responsável.

Figura 2 - Radiografia panorâmica

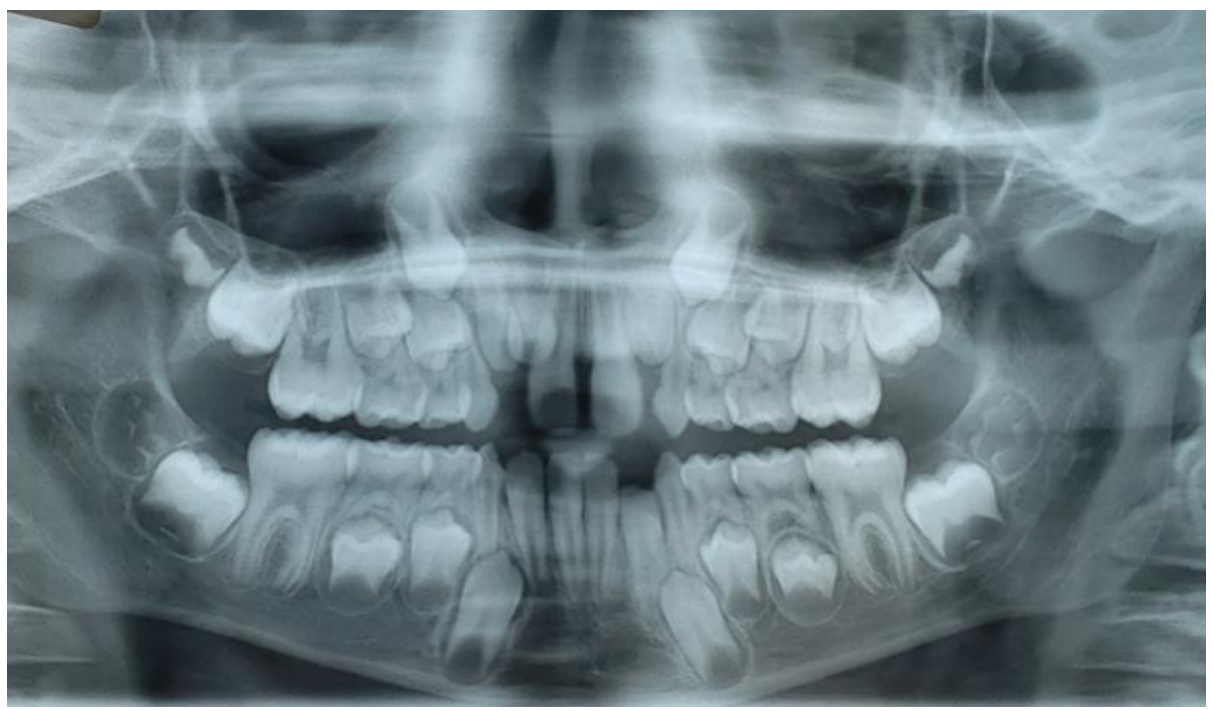

Fonte: Acervo pessoal.

Figura 3 - Telerradiografia de perfil 


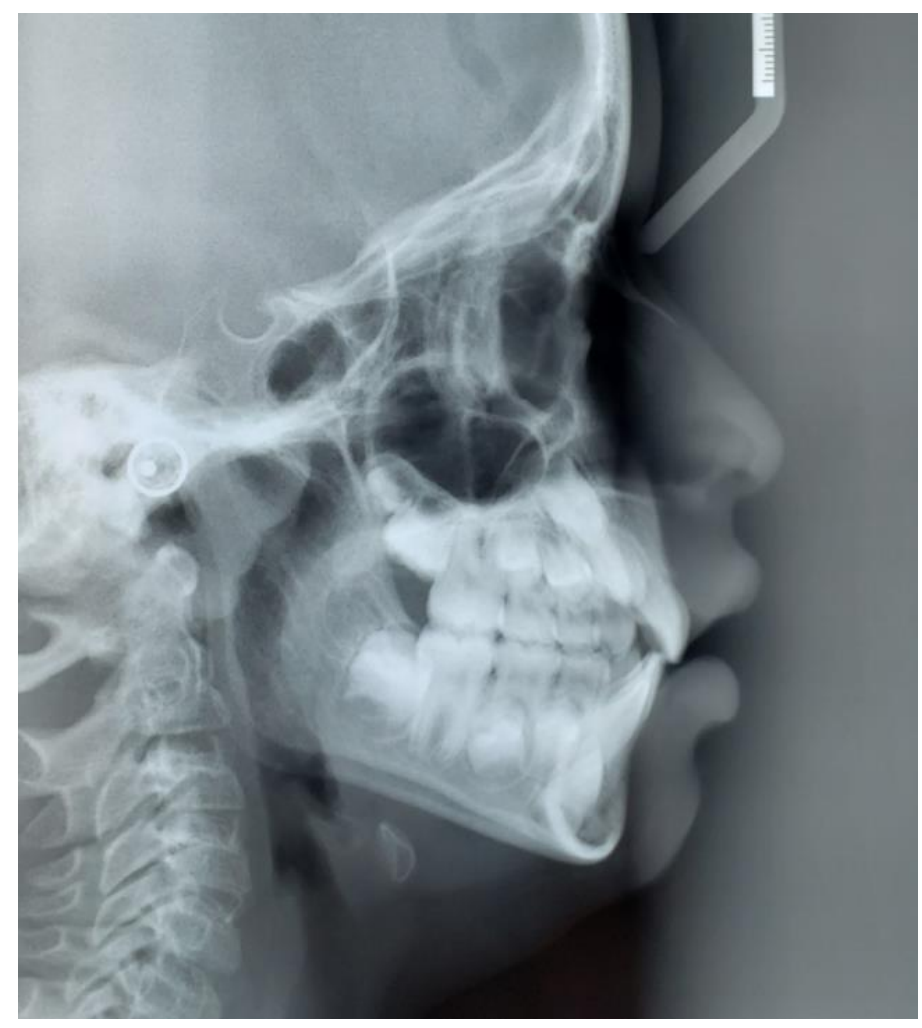

Fonte: Acervo pessoal.

O tratamento de escolha na primeira fase, foi a correção da atresia maxilar, e, consequente mordida cruzada, a qual foi observada no desvio da mandíbula para a esquerda em $\mathrm{MIH}$, caracterizando uma mordida cruzada unilateral funcional, além de proservação do elemento supranumerário, para definição do melhor momento de sua exodontia. Para essa fase, optou-se pelo aparelho Mcnamara. (Figuras 4 e 5) Podemos observar, na figura 4, 0 reposicionamento mandibular com correção da linha média inferior.

O protocolo de ativação do aparelho Mcnamara, inicialmente foi de 2/4 de volta pela manhã e 2/4 de volta à noite, durante 7 dias consecutivos, antecedentes à próxima consulta.

Figura 4 - Fotografia frontal pós-expansão. 
Pereira FVA

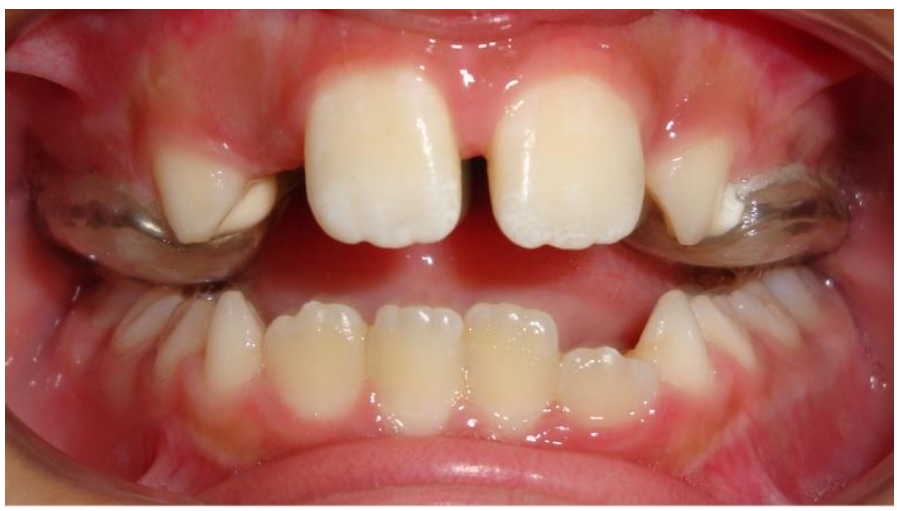

Fonte: Acervo pessoal.

Após 3 meses à essa ativação, uma radiografia oclusal foi solicitada para observação da neoformação óssea na sutura palatina mediana. (Figura 5)

Figura 5 - Radiografia oclusal pós-ativação.

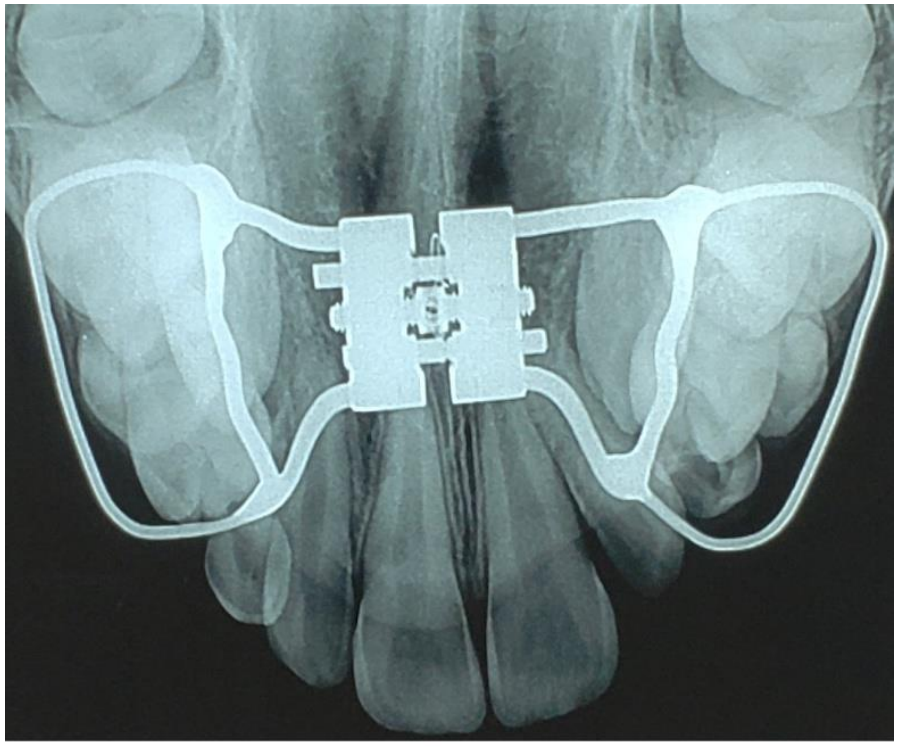

Fonte: Acervo pessoal. 
O protocolo não foi atingido, pois a responsável teve dificuldades de realizar todas as ativações. Após remoção do Mcnamara observou-se a necessidade de um pouco mais de expansão, o que foi realizado com uma Placa de Hawley com expansor. Ativação de $1 / 4$ de volta 2 vezes por semana. A TC estava planejada para a fase posterior à expansão (pré-cirúrgica), contudo, o elemento supranumerário irrompeu por palatina, conforme mostra a figura 6 , eliminando assim, sua necessidade.

Figura 6 - Fotos após a remoção da Placa de Hawley com expansor:

A) vista lateral direita, B) vista frontal, C) vista oclusal.

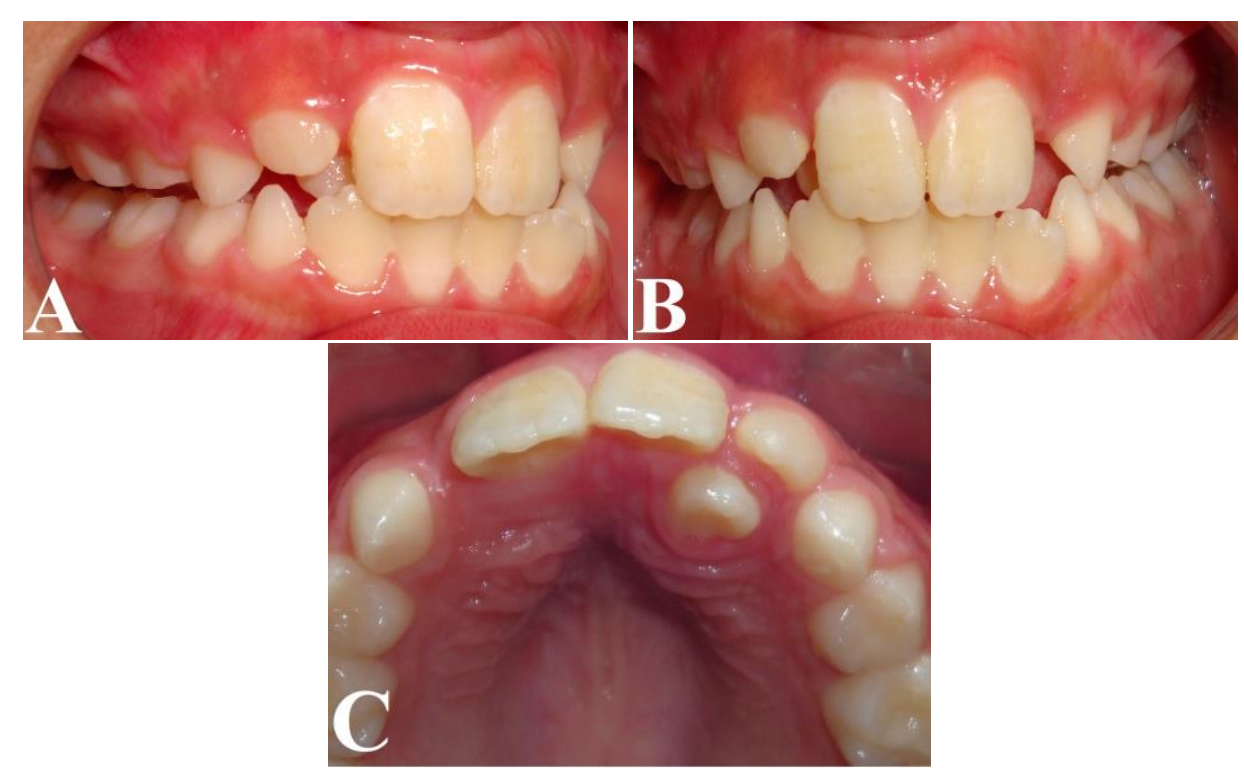


Fonte: Acervo pessoal.

A partir daí, foi solicitada a exodontia do elemento supranumerário. $\mathrm{Na}$ figura 7 observamos a exodontia já realizada. Nesse momento, foi removido o aparelho e dado alta provisória para o paciente.

Figura 7 - Após remoção cirúrgica do supranumerário: foto oclusal.

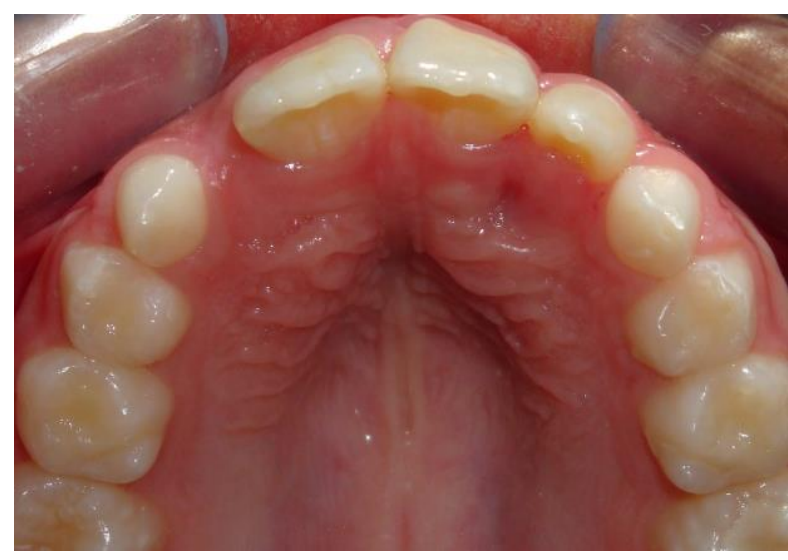

Fonte: Acervo pessoal.

\section{DISCUSSÃO}

Os dentes supranumerários são os dentes considerados sobressalentes na arcada dentária de um paciente. Essa anomalia não é equilibrada ou compensada na arcada dentária considerada normal (PRIMOSCH, 1981).

Estudos feitos por (ROCHA A.M.L. et al., 2002) mostraram que os supranumerários têm seu aparecimento ligado a diversos fatores genéticos, como displasia cleidocranial e as síndromes hereditárias relacionadas à hiperdontia, tais como a angio osteohipertrofia, sendo corroborado por (FOLWARCZNA; RUSSEL, 2003) que o desenvolvimento dessa anomalia tem sua origem praticamente desconhecia, porém em geral está ligada a alguns fatores genéticos.

Eles podem surgir tanto na dentição permanente como na dentição decídua. Muitas das vezes permanecem ocultos e em outros casos podem ser identificados a partir do surgimento da dentição. Eles podem estar com posição 
ectópica ou invertida, podem aparecer na mandíbula, na maxila ou em ambos e ainda aparecem isolada, múltipla, bi ou unilateral (REIS et al., 2006).

Os supranumerários poderiam ser a reaparição da dentição não mais utilizada, mas que esteve presente com os ancestrais. Outra explicação seria a hiperatividade da lâmina dentária, ou ainda, seria decorrente de fatores locais, como inflamação, cicatrização, entre outras explicações desse fenômeno (REIS et al., 2006).

Em geral, o desenvolvimento de um supranumerário acontece em pacientes masculinos em uma proporção 2:1, quando comparado ao sexo feminino. E ainda acomete, cerca de cinco vezes mais a dentição permanente do que a decídua. E ocorre de $0,15 \%$ a $3,7 \%$ na população, sendo mais comumente encontrada em pacientes com até 20 anos de idade (CORRÊA, et al. 2009).

Os dentes supranumerários tem sua classificação de acordo com a sua localização. Os paramolares são aqueles que irrupcionam por vestibular, lingual ou interproximal e os distomolares são os posteriores ao terceiro molar. Os mesiodentes ou mesiodens são os localizados na linha da pré-maxila ou maxila (COSTA; FACCO; NUNES, 2003). Em geral, os supranumerários são encontrados na região superior anterior e geralmente causam inquietações ao paciente e seus familiares, especialmente pelo retardo na irrupção da dentição no local, ou ainda pelos aspectos funcionais e estéticos com distintas alterações (NEGRETE, 2008).

A descoberta de um dente supranumerário pode ser feita de uma forma antecipada ou através da sua irrupção. Pela forma antecipada é feita através de uma radiografia panorâmica ou de exames de rotina que possam detectar essa anomalia. Além disso, podemos utilizar duas técnicas radiográficas para identificação dos supranumerários que são a técnica de Clark e a técnica oclusal. A técnica de Clark é utilizada na maxila e a técnica oclusal na mandíbula. Através da técnica de Clark, usamos duas posições diferentes, uma periapical ortorradial 
e outra mesio ou distorradial. Esta técnica pode nos ajudar a estimar a localização exata de dentes, especialmente em casos de mesiodens impactados (GIOVANETTI et al. 2016).

Quando os supranumerários são descobertos antes da irrupção, é um tratamento simples e que não tem complicações na arcada dentária ou especialmente diminui ou neutraliza a possibilidade do aparecimento da má oclusão (ALMEIDA et al., 1997).

Supranumerários podem ser identificados de forma precoce, no início da infância, entre os 5 e 7 anos de idade. A realização do procedimento quanto mais antecipada, menores serão as sequelas. Caso contrário, algumas consequências funcionais e estéticas aparecerão, como a erupção tardia do incisivo permanente, retenção dentária, inclinações, rotações, diastemas e impacções, entre outros (MEZZOMO; TONELOTTO; MUNDSTOCK, 2004).

É preciso um bom acompanhamento clínico junto ao paciente. Quando as implicações forem aumentadas ou intensificadas, certamente o melhor caminho é a extração cirúrgica desse supranumerário evitando assim, maiores complicações ao paciente. Existem efeitos desagradáveis ao paciente, o que o leva ao consultório odontológico para a realização do tratamento que seja eficaz ao seu caso (CACHAPUZ; SIQUEIRA; PRIETSCH, 2002).

Com a evolução da tecnologia, hoje já é possível a utilização de uma tomografia computadorizada em 3D que é uma nova forma de avaliar os dentes impactados e supranumerários com informações importantes, que antes não eram possíveis com a radiografia oclusal e a radiografia panorâmica (REIS et al., 2006).

São diversas as formas de tratamento, porém existem controvérsias sobre o assunto. Alguns autores sugerem a extração cirúrgica logo após um minucioso diagnóstico da anomalia, bem como, (ROTBERG; KOPEL, 1984) que recomendaram a extração do supranumerário no momento que foi detectado para prevenir o tratamento ortodôntico futuro e minimizar a perda óssea. Eles alegaram que a idade ótima para a remoção cirúrgica do supranumerário foi 
antes dos 5 anos. Já outros pesquisadores, dizem que é necessário que o paciente seja observado, e escolhido o melhor momento para a extração cirúrgica (CORRÊA, 2009).

Segundo (VALARELLI et al., 2012) quanto antes à realização do procedimento cirúrgico ou do acompanhamento clínico ao paciente, consequentemente menores serão as sequelas para o mesmo. Os estudos feitos por (MARCHETTI; OLIVEIRA, 2015; GIOVANETTI K et al., 2016; ISSAO; KAHTALIAN, 1968) mostram que, quando as implicações forem intensificadas ou aumentadas, recomenda-se que a extração cirúrgica seja então o melhor caminho. Com isso, pode-se evitar maiores complicações ao paciente. É preciso que se avalie caso a caso, pois nem sempre se tem uma avaliação anterior à erupção dos mesiodentes. Alguns efeitos desagradáveis levam os pacientes ao consultório odontológico para a realização do tratamento que seja eficaz ao seu caso (VALARELLI et al., 2012).

A extração e um tratamento precoce, ainda são os melhores métodos para essa anormalidade, existem soluções para o tratamento, posterior ao aparecimento do supranumerário, mas isso, além de gerar custo também pode gerar dores ao paciente. Existem diferentes condutas para os supranumerários, tais como, a não interferência no processo fisiológico natural da erupção, a remoção precoce e suas desvantagens, a remoção e o tratamento ortodôntico e a remoção antes da instalação da má oclusão (FERREIRA et al., 2011). A remoção cirúrgica de um dente supranumerário é recomendada, quando este estiver interferindo na cronologia normal de erupção dos demais dentes adjacentes, podendo assim, realizar uma abordagem mais conservadora (HUANG,1992). O pós-tratamento é de suma importância para o sucesso e satisfação do paciente, portanto, a contenção e acompanhamento, tem papel fundamental (CAL NETO; CUNHA; MIGUEL, 2002).

\section{CONCLUSÃO}


Uma vez diagnosticados os dentes supranumerários, sejam eles individuais ou múltiplos, as decisões com relação à escolha do tratamento, precisam ser analisadas de acordo com cada caso, respeitando suas individualidades. No relato clínico exposto, observamos uma decisão de exodontia posterior à ERM, onde o elemento supranumerário não obliterava a linha de irrupção dos elementos permanentes. Com a irrupção desse elemento, foi efetivado um ato cirúrgico mais simples, diminuindo riscos e complicações, principalmente em um paciente com idade pediátrica, além de evitar uma grande exposição aos raios $\mathrm{X}$, com a realização de uma Tomografia Computadorizada.

\section{REFERÊNCIAS}

ALMEIDA, R.R. et al. Supranumerários- Implicações e procedimentos clínicos. Revista Dental Press de Ortodontia e Ortopedia Maxilar, v.2, n.6, p. 91-108, 1997.BEZERRA, P.K.M.; BEZERRA, O.M.; CAVALCANTI, A.L. Dentes supranumerá

rios: revisão da literatura e relato de caso. Revista de Ciências Médicas e Biológicas, v.6, n.3, p.349-56, 2007.

CACHAPUZ, P.F.; SIQUEIRA, F.S.; PRIETSCH, J.R. Tratamento ortodôntico interceptativo de maloclusão causada por mesiodente: relato de caso. Revista da Faculdade de Odontologia de Porto Alegre, v.43, n.1, p.18-22, 2002.

CAL NETO, J.O.A.P.; CUNHA, D.L.; MIGUEL, J.A.M. Diastemas interincisais superiores associados a dentes supranumerários-considerações clínicas e relato de caso. Jornal Brasileiro de Ortodontia e Ortopedia Facial, v.7, n.39, p.23944, 2002.

COLUMBANO NETO, J.; ROCHA, A.M.L.; SOUZA, M.M.G. Hiperdontia na região de incisivos superiores. Jornal Brasileiro de Ortodontia e Ortopedia Facial, v.7, n.41, p.389-96, 2002.

CORRÊA, F.G. et al. Prevalência de dentes supranumerários- estudo retrospectivo. International Journal of Dentistry, v.8, n.1, p.11-5, 2009.

COSTA, S.J.M.; FACCO, E.S.; NUNES, E.L. Distomolares. Revista da Faculdade de Odontologia de Lins, v.15, n.1, p.33-6, 2003.

FERREIRA, R.A.; SILVA, E.R.; AZENHA, M.R. Achados clínicos e radiográficos de dente supranumerário na região posterior da maxila. Revista da Academia Tiradentes de Odontologia, v.11, n.1, p.253-60, 2011. 
FOLWARCZNA, M.A.; RUSSELL, K.A. Mesiodens- Diagnosis and Managementof a common supernumerary tooth. Journal of Canadian Dental Association, v.69, n.6, p.362-6, 2003.

GIOVANETTI K. et al. Mesiodens. A case report. Revista Faculdad de Odontologia Universidad Antioquia. v. 28, n. 1, p. 210-9, 2016.

HENRIQUES J.F.C. et al. Expansão Rápida da Maxila (ERM) Associada à Irrupção de Mesiodens e Tração Cirúrgica do dente 21. Revista Clinica Ortodontia Dental Press, v. 7, n.5, np, 2008.

HUANG, W.H., TSAI, T.P., SU, H.L Mesiodens in lhe primary dentition stage: a radiographic study. Journal Dentistry for Chíldren., Chicago, v.59, n.3, p.186189, 1992.

ISSÁO, M.; KAHTALIAN, L. Y. Dentes Supranumerários na região Anterosuperior mista. Revista de Odontologia da Faculdade de São Paulo, v. 6, n. 2, p.137-150, 1968.

MARCHETTI G.; OLIVEIRA R.V. Mesiodens - Dentes Supranumários: Diagnostico causas e Tratamento. Revista UNINGÁ Review, v. 24, n. 1, p. 1923, 2015.

MEZZOMO, C.S.; TONELOTTO, P.R.; MUNDSTOCK, C.A. Diagnóstico tardio de mesiodens e sua relação com mal oclusão: relato de caso. Revista da Faculdade de Odontologia de Porto Alegre, v.45, n.1, p.41-3, 2004.

NEGRETE, D. Estudo da prevalência de dentes supranumerários em pacientes indicados ao tratamento ortodôntico através de radiografias panorâmicas. 2008. (dissertação) - Universidade Cidade de São Paulo, São Paulo, 2008.

PRIMOSCH, R.E. Anterior supernumerary-teeth - assessment and surgical intervention in children. Pediatric Dentistry Journal, v.3, n.2, p.204-15, 1981.

REIS, L.F.G. et al. Dentes supranumerários retidos interferindo no tratamento ortodôntico. RSBO (impresso), v. 3, n. 2, p.20-26, 2006.

ROTBERG S., KOPEL H. Early versus late removal of mesiodens: a clinical study of 375 children. Compendium of Continuing Education in Dentistry, v. 2, p.115-120. 1984.

VALARELLI, F.P., et al. Correção ortodôntica de pacientes com mesiodens. Revista UNINGÁ, v., 33, n. 1, p.5-16, 2012. 